\title{
First-order transitions and the performance of quantum algorithms in random optimization problems
}

\author{
Thomas Jörg, ${ }^{1}$ Florent Krzakala, ${ }^{2,3}$ Guilhem Semerjian, ${ }^{1}$ and Francesco Zamponi ${ }^{4,1}$ \\ ${ }^{1}$ LPTENS, CNRS UMR 8549, associée à l'UPMC Paris 06, 24 Rue Lhomond, 75005 Paris, France. \\ 2 ESPCI ParisTech, CNRS UMR 7083 Gulliver, 10 rue Vauquelin, 75005 Paris, France \\ ${ }^{3}$ T-Division and Center for Nonlinear Studies, Los Alamos National Laboratory, NM 87545 USA \\ ${ }^{4}$ Princeton Center for Theoretical Science, Princeton University, Princeton, NJ 08544, USA
}

\begin{abstract}
We present a study of the phase diagram of a random optimization problem in presence of quantum fluctuations. Our main result is the characterization of the nature of the phase transition, which we find to be a first-order quantum phase transition. We provide evidence that the gap vanishes exponentially with the system size at the transition. This indicates that the Quantum Adiabatic Algorithm requires a time growing exponentially with system size to find the ground state of this problem.
\end{abstract}

PACS numbers: 75.10.Nr; 03.67.Ac; 64.70.Tg

Theoretical research on quantum computing is motivated by the exciting perspective of computers that take intrinsically advantage of the laws of quantum mechanics. Besides the great effort of research towards the physical realization of these devices, a lot of activity has been devoted to the development of algorithms that could use quantum properties to achieve a faster velocity in performing computational tasks with respect to classical devices. A typical problem that is encountered in almost all branches of science is that of optimizing irregularly shaped cost functions: the Quantum Adiabatic Algorithm (QAA) [1, 2] is in principle able to tackle such problems in a universal way. Suppose one wishes to find the ground state of a Hamiltonian $H_{P}$ acting on $N$ qubits. To perform the QAA one considers a simpler Hamiltonian $H_{Q}$, such that the quantum computer can be easily initialized in its ground state. If one slowly interpolates the Hamiltonian $H(t)$ of the quantum computer from $H_{Q}$ to $H_{P}$, the adiabatic theorem ensures that, with high enough probability, the system will remain at all times in the ground state of the interpolating Hamiltonian. Hence, at the end of the evolution, it will be in the ground state of $H_{P}$ and the original problem will be solved. The crucial question is of course, how slow the evolution should be in the thermodynamic limit $N \rightarrow \infty$. Quite generally, the adiabaticity condition requires the rate of change of $H(t)$ to be smaller than the (squared) gap between the ground state and the first excited state of $H(t)$. Hence, the time needed to ensure adiabaticity will diverge in the thermodynamic limit whenever a quantum phase transition, at which the gap is expected to vanish [3], is encountered during the interpolation between $H_{Q}$ and $H_{P}$. It is well established that the gap vanishes at least polynomially in $N$ at a quantum secondorder critical point [3] (except in some cases in presence of disorder [4]), while it vanishes exponentially in $N$ at a first-order phase transition [5-7]. First-order phase transitions are thus particularly dangerous for the QAA.

The formal computational complexity theory classifies the difficulty of a problem according to a worst-case criterion. It might, however, well be that "most" of the problems in a given class are easy, even though a few atypical instances are very difficult. To give a precise content to this notion of typicality the research has turned to the study of random instances, defining a probability distribution on the space of instances. Statistical mechanics tools have provided a very detailed and intricate picture of the properties of the configuration space of such typical problem Hamiltonians $H_{P}[\underline{8}]$. Random instances were also used to benchmark the performance of the QAA, and early results generated considerable excitement by reporting polynomial scaling of the minimum gap for sizes up to $N \sim 100$ [2, 9]. However, some evidence of the presence of first-order phase transitions has been recently reported [5 7], which is natural from the point of view of mean-field quantum spin glasses [10]. These studies rely on numerical investigations of small systems $(N \lesssim 256)$ and/or on perturbation theory close to $H_{P}$, hence they should be completed by a non-perturbative analytic treatment in the thermodynamic limit.

This is what we achieve in this Letter, reporting the results of the first analytical study of a random, quantum, finite-connectivity optimization problem (namely, random regular 3-XORSAT in a transverse field), that is believed to be largely representative of the generic behavior of these problems. We compute the complete phase diagram of the model in the thermodynamic limit $N \rightarrow \infty$ and for a uniformly random distribution of instances, as a function of temperature and transverse field. This is possible thanks to the quantum cavity method, recently introduced in [11] and further developed in [12] (see [13] for related work), that allows to solve exactly these problems by generalizing to the quantum case the method developed for classical models [14]. Our main result is the occurrence of a first-order phase transition at zero temperature as a function of the transverse field. We corroborate the analytical results with Exact Diagonalization and Quantum Monte Carlo data. We provide evidence 


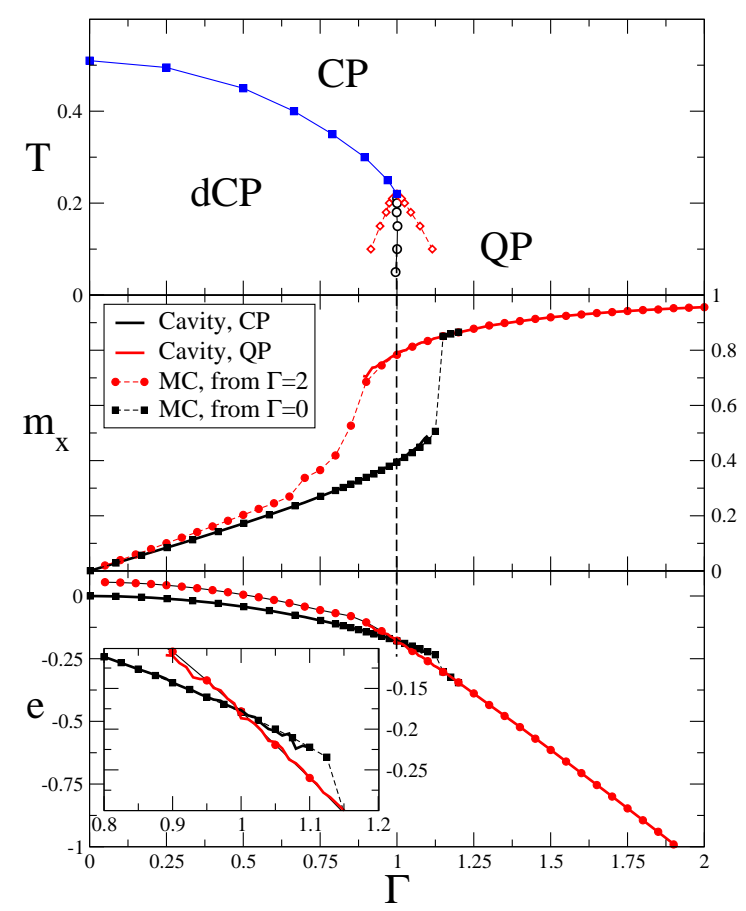

FIG. 1: (Top) Phase diagram of Eq. (1) for $c=3$. Open symbols are results of the RS calculation: first-order transition line separating the CP and QP (circles), with the corresponding spinodals (diamonds). Full symbols are the result of the 1RSB calculation: squares, clustering transition separating the CP and dCP. (Bottom) Energy and $m_{x}$ as a function of $\Gamma$ for temperature $T=0.05$. MC data for a sample with $N=2049$.

that the gap vanishes exponentially in the size of the system at the transition. These results strongly suggest that the QAA requires an exponentially large time in $N$ to find the ground state of this problem.

Methods. - We focus on the random c-regular 3XORSAT problem [15], that in spin language is defined by the Hamiltonian

$$
H=H_{P}+H_{Q}=\sum_{a=1}^{M}\left(1-J_{a} \sigma_{i_{1}^{a}}^{z} \sigma_{i_{2}^{a}}^{z} \sigma_{i_{3}^{a}}^{z}\right)-\Gamma \sum_{i=1}^{N} \sigma_{i}^{x} .
$$

Here, $J_{a}= \pm 1$ with equal probability. The 3 spins $i_{1}^{a}, i_{2}^{a}, i_{3}^{a}$ involved in clauses $a=1, \cdots, M=N c / 3$ are chosen uniformly at random among all possible choices such that each spin enters exactly in $c$ clauses. In the classical limit $\Gamma=0$, a given instance of the system is called satisfiable (SAT) if there is a ground state of zero energy, UNSAT otherwise.

The thermodynamic properties of the model in the thermodynamic limit $N \rightarrow \infty$, and on average over the disorder, can be obtained by means of the cavity method [14]. For quantum models, the cavity method allows to reduce the solution of the model to the problem of finding the fixed point of a functional equation for the local spin effective actions [11, 12] (this functional equa-

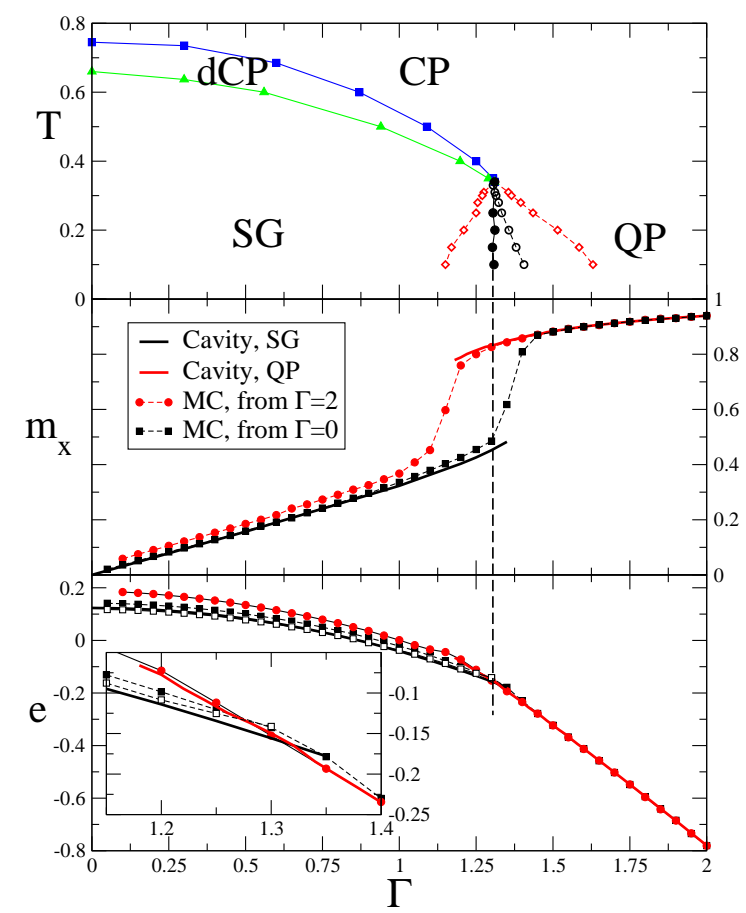

FIG. 2: (Top) Phase diagram of Eq. (11) for $c=4$. Symbols as in Fig. 1 with the addition of the spin-glass transition line (full triangles); the correct first-order transition line is obtained from the 1RSB calculation (full circles). (Bottom) Energy and $m_{x}$ as a function of $\Gamma$ for temperature $T=0.05$. MC data for $N=120$ and averaged over 20 samples (full symbols) and extrapolated in $1 / N$ to the $N \rightarrow \infty$ limit (open symbols). Black curve, starting from the classical ground state found using an exact MAXSAT solver [17]. Red curve, starting from the QP.

tion being solved numerically as in 12] with a population dynamics algorithm [14]). In presence of one single pure state, the method discussed in [11, 12], that goes under the name of replica symmetric (RS), is enough to obtain the correct solution. However, in order to describe the low-temperature glassy phase, which is characterized by a large number of pure states, one has to introduce a generalization of the RS cavity method that goes under the name of one-step replica symmetry breaking (1RSB) [14]. This generalization, that we introduce here for the first time in the context of quantum diluted models, proceeds along the line of the classical computation (see [15] for the solution of (11) at $\Gamma=0$ ) using as variables the imaginarytime spin trajectories as detailed in [12]. Additionally, for finite $N$, we performed Exact Diagonalization (ED) using the Ritz functional method [16], and Quantum Monte Carlo (QMC) simulations using the heat-bath algorithm introduced in [12].

Results of the cavity method. - In the classical limit 15] when $\Gamma=0$, the model is SAT (with a probability going to 1 as $N \rightarrow \infty$ ) for $c<3$, UNSAT for $c>3$, while in the marginal case $c=3$ it is SAT with finite proba- 


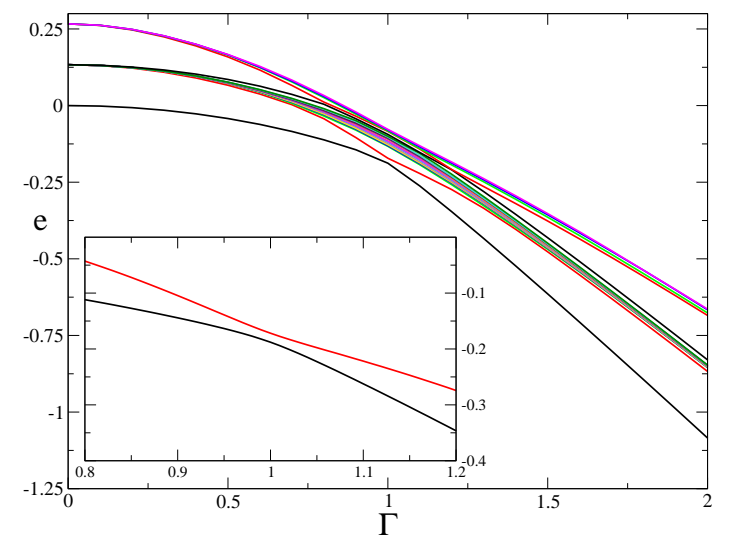

FIG. 3: Lowest energy levels from exact diagonalization of a USA instance with $c=3$ and $N=15$. In the inset the region close to the phase transition is magnified.

bility. Let us begin the description of our results with the simpler case $c=3$. The RS computation predicts, at low enough temperature $T \lesssim 0.24$, a first-order transition between two different paramagnetic $\left(m_{z}=\left\langle\sigma_{i}^{z}\right\rangle=0\right)$ phases: the Classical Paramagnet (CP) characterized by a small value of transverse magnetization $m_{x}=\left\langle\sigma_{i}^{x}\right\rangle$, and the Quantum Paramagnet (QP) that has a larger value of $m_{x}$. This transition and the corresponding spinodals are shown in the $(\Gamma, T)$ phase diagram of the top panel in Fig. 1. the transition is found around $\Gamma_{\mathrm{c}}(T) \approx 1.0$ for all values of $T \lesssim 0.24$. We also report in the bottom panel of Fig. 1 the cavity method predictions for $m_{x}$ and the energy density $e=\langle H\rangle / N$ at very low temperature. The outcome of the 1RSB computation is twofold: it confirms that the RS computation of the thermodynamic observables is in this case correct in the whole phase diagram $(\Gamma, T)$, in particular they are singular only on the RS transition line. Moreover it unveils that, for low enough values of $T$ and $\Gamma$ the CP phase is actually a "dynamical CP", in technical terms a 1RSB phase with Parisi breaking parameter $x$ equal to 1 , where an exponential number of pure states coexist. The attribute dynamical, taken from the literature on classical mean-field spin glasses and optimization problems [8], emphasizes that equilibrium thermodynamic properties are unaffected as one crosses the line between $\mathrm{CP}$ and $\mathrm{dCP}$ (also plotted in Fig. 1).

We turn now to the $c=4$ case (which is representative of the behavior for any $c>3$ ), for which the results are displayed in a similar fashion on Fig. 2. It has a richer phenomenology very similar to the one of fully-connected mean-field models [10]. Indeed the dCP undergoes a thermodynamically second-order phase transition to a true Spin-Glass (SG) phase (with a sub-exponential number of pure states and $x<1$ ). At low enough temperature the thermodynamic transition becomes first order, between the 1RSB SG and the QP. For this reason, the RS computation gives a wrong result for the first-order

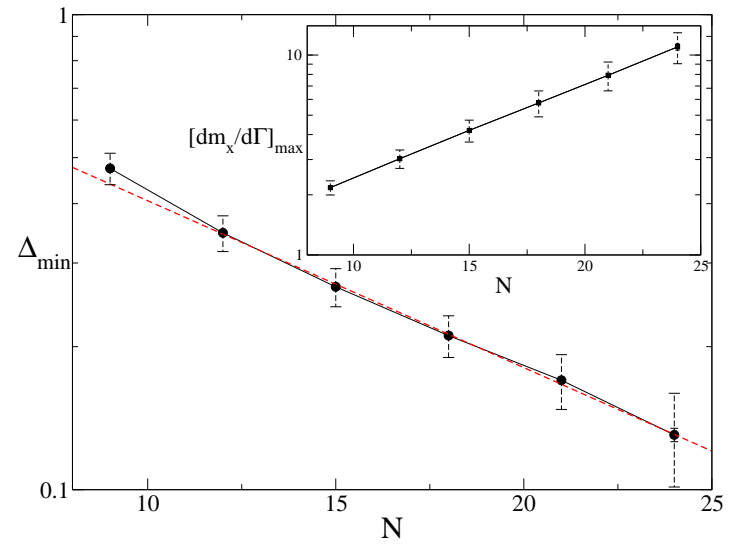

FIG. 4: Data for $c=3$ on USA instances. (Main panel) Average of the minimal gap $\Delta_{\min }$ as a function of $N$. Dashed line is a fit to $\Delta_{\min }(N)=0.911 \exp (-0.081 N)$. Inset: average of $\left[d m_{x} / d \Gamma\right]_{\max }$. In both cases, error bars are of the order of the symbol size except when explicitly shown $(N=24)$. Dashed bars represent the standard deviation of a single realization of the random variables $\Delta_{\min }$ and $\left[d m_{x} / d \Gamma\right]_{\max }$.

transition line, see top panel of Fig. 2. In both cases we conclude on the existence of a first-order quantum phase transition at $\Gamma=\Gamma_{\mathrm{c}}$ and zero temperature, separating the dCP (for $c=3$ ) or the SG (for $c=4$ ) from the QP. The transition extends in a line $\Gamma_{\mathrm{c}}(T)$ at low enough temperature, which is almost independent of $T$.

Numerical investigations. - A first instructive example of the relevance of this transition is found by comparing the cavity results with QMC (Figs. 1 2 lower panel). We first run a QMC starting from the classical ground state at $\Gamma=0$ and slowly increasing $\Gamma$. For $c=3$, instances have a finite probability of being SAT, and otherwise have an energy of order $1 / N$ (see below and [15]): since SAT instances can be solved in polynomial time using the Gauss elimination algorithm, we can limit ourselves to the study of this simple case, and we can then run the QMC for very large sizes $(N=2049)$. For $c=4$, the problem is typically UNSAT [15], finding the ground state is very hard (actually, NP-hard), and we are limited to much smaller sizes $(N \leq 120)$; yet a good extrapolation in $1 / N$ to the thermodynamic limit is possible. In both cases we find that the QMC data follow closely the cavity result for the dCP or SG phases, respectively. As expected for a first-order transition, we find some hysteresis around $\Gamma_{\mathrm{c}}$ before the system finally jumps to the QP phase. We then consider a more interesting QMC run starting from large $\Gamma=2$ in the QP phase and slowly decreasing $\Gamma$. For both $c=3,4$, QMC data follow the cavity ones down to the transition, but in both cases, the energy remains extensively higher than the ground-state energy for any $\Gamma<\Gamma_{\mathrm{c}}$. This is already an important indication of the difficulty in finding the ground state in the $\mathrm{dCP}$ and SG phases, even in presence of quantum fluctuations. 
We have further investigated the consequences of the transition at $\Gamma_{\mathrm{c}}$ for the QAA by investigating with exact diagonalization tools the dependence on $\Gamma$ of the low-energy part of the spectrum of $H$ for small sizes. To unambiguously define the gap $\Delta(\Gamma)$ between the ground state of $H$ and its first excited state at all values of $\Gamma$, we concentrated on instances of $H_{P}$ having a Unique Satisfying Assignment (USA), i.e., a single classical ground state. For $c=3$ and $N \rightarrow \infty$, the fraction of SAT and USA instances are $f_{\mathrm{SAT}}=0.609 \pm 0.003$ and $f_{\text {USA }}=0.2850 \pm 0.0022$, as determined by using a DavisPutnam-Logemann-Loveland-like algorithm to count the number of solutions of 40000 instances [18]. Since USA instances are a finite fraction of the uniform ensemble, they can be easily constructed. The spectrum of a typical USA instance of $N=15$ spins is reported in Fig. 3. We observe, as expected, that the gap $\Delta(\Gamma)$ has a minimum $\Delta_{\text {min }}$ close to the phase transition at $\Gamma_{\mathrm{c}}$ (recall that $\Gamma_{\mathrm{c}} \approx 1$ for $c=3$ at $\left.N \rightarrow \infty\right)$. Around the same $\Gamma_{\mathrm{c}}, m_{x}$ changes abruptly, hence its derivative has a large maximum $\left[d m_{x} / d \Gamma\right]_{\max }$. In Fig. 4 we show the behavior of the average $\Delta_{\min }$ and $\left[d m_{x} / d \Gamma\right]_{\max }$ as a function of $N$. Our data are clearly consistent with an exponential scaling of the gap, which is expected in presence of a firstorder transition (see [5] for a discussion on how to compute the prefactor in the exponential in fully-connected models), and an exponential divergence of $\left[d m_{x} / d \Gamma\right]_{\max }$. The probability distribution over instances of $\Delta_{\min }$ and $\left[d m_{x} / d \Gamma\right]_{\max }$ has a unique peak close to their average, and its variance is also reported in Fig. 4 (dashed bars). This shows that all instances undergo a first order transition of the same kind in the thermodynamic limit.

Let us finally suggest that the main differences between our observations and the ones of [6, 9] arises from the method of construction of instances. Most random optimization problems that undergo a SAT-UNSAT transition as a control parameter is continuously tuned still have an exponential number of ground states right before they become UNSAT. Conditioning on USA instances is thus, contrary to the case studied in this Letter, an exponentially rare event which restricts the study to extremely atypical instances and that also forbids the construction of large instances. There exists however a natural family of difficult optimization models (e.g., Eq. (1) with $c=4$ and $J_{a}=1 \forall a$ ) which have unique ground states with probability 1 in the thermodynamic limit [19]. This is thus a practical way of generating large USA instances as typical ones in a uniform random ensemble.

Conclusions. - We have obtained the full phase diagram of the quantum regular XORSAT optimization problem as a function of $T$ and $\Gamma$. Our main results are: i) There is a first-order quantum phase transition at $T=0$ between a Paramagnetic or a Spin-Glass phase and a Quantum Paramagnetic phase, at a critical value of $\Gamma=\Gamma_{c}$; ii) The transition is due to a crossing between the low- $\Gamma$ classical-like ground state, and the high-
$\Gamma$ quantum paramagnetic state. It is of very different nature from the level crossing at infinitesimal $\Gamma$ between different spin-glass ground states discussed in [6] ; iii) The first-order transition is observed for almost all instances, even for very small $N$; iv) The transition is associated to an exponentially vanishing gap of $H$, hence the Quantum Adiabatic Algorithm requires a run time scaling exponentially with system size. These results indicate that quantum adiabatic computations, at least in their original formulation [1, 2], fail for difficult optimization problems.

The method introduced here is not restricted to XORSAT, and can be applied to investigate other random optimization problems, such as, e.g., the exact cover discussed in [6, 7, 9] or random $K$-SAT. Another closely related problem is MAP decoding of LDPC codes. The study of these problems should be very interesting, since in the classical case they typically have exponentially many solutions even at the SAT-UNSAT transition. This huge degeneracy will be partially lifted by adding a transverse field, but we expect the low energy spectrum of these problems to be quite complicated, and characterized by many almost-degenerate low energy states. What is the precise definition of the relevant gap for the QAA in these cases is clearly an interesting problem. It is, however, rather natural, based on the accumulated knowledge on classical optimization problems [8] and meanfield spin-glasses [5, 10], to believe this first-order transition, and thus the failure of the QAA to be a generic feature in these problems. Yet, the instances that are encountered in practical applications are often very different from the random instances investigated here: the latter are characterized by a locally tree-like graph, while the former have more structure. Extending these results to more realistic instances is an important direction for future research.

We wish to thank B. Altshuler, J. Roland and A. P. Young for illuminating discussions.

[1] B. Apolloni, N. Cesa-Bianchi and D. de Falco, in Proceedings of the 1988 Ascona-Locarno Conference, 97, World Scientific (1990); T. Kadowaki and H. Nishimori, Phys. Rev. E 58, 5355 (1998)

[2] E. Farhi et al., Science 292, 472 (2001)

[3] S. Sachdev, Quantum Phase Transitions, CUP (1999).

[4] D. S. Fisher, Phys. Rev. B 51, 6411 (1995).

[5] T. Jörg et al., Phys. Rev. Lett. 101, 147204 (2008).

[6] M.H.S. Amin and V. Choi, Phys. Rev. A 80, 062326 (2009); B. Altshuler et al., arXiv:0908.2782 E. Farhi et al., arXiv:0909.4766.

[7] A.P. Young, S. Knysh, V. N. Smelyanskiy, Phys. Rev. Lett. 104, 020502 (2010).

[8] R. Monasson and R. Zecchina, Phys. Rev. E 56, 1357 (1997); M. Mézard, G. Parisi and R. Zecchina, Science 297, 812 (2002); F. Krzakala et al., Proc. Natl. Acad. Sci. 
104, 10318 (2007); M. Mézard and A. Montanari, Information, Physics and Computation, Oxford University Press (2009).

[9] T. Hogg, Phys. Rev. A 67, 022314 (2003); A.P. Young, S. Knysh and V.N. Smelyanskiy, Phys. Rev. Lett. 101, 170503 (2008).

[10] Y. Y. Goldschmidt, Phys. Rev. B 41, 4858 (1990); T. M. Nieuwenhuizen and F. Ritort, Physica A 250, 8 (1998); G. Biroli and L.F. Cugliandolo, Phys. Rev. B 64, 014206 (2001); L.F. Cugliandolo, D.R. Grempel and C.A. da Silva Santos, Phys. Rev. B 64, 014403 (2001).

[11] C. Laumann, A. Scardicchio and S.L. Sondhi, Phys. Rev. B 78, 134424 (2008).

[12] F. Krzakala et al., Phys. Rev. B 78, 134428 (2008).
[13] M. B. Hastings, Phys. Rev. B 76, 201102(R) (2007); S. Knysh and V.N. Smelyanskiy, Phys. Rev. E 78, 061128 (2009); E. Bilgin and D. Poulin, Phys. Rev. B 81, 054106 (2010).

[14] M. Mézard and G. Parisi, Eur. Phys. J. B 20, 217 (2001).

[15] S. Franz et al., Europhys. Lett. 55, 465 (2001); S. Franz et al., Phys. Rev. Lett. 87, 127209 (2001).

[16] T. Kalkreuter and H. Simma, Comput. Phys. Commun. 93, 33 (1996).

[17] http://www.laria.u-picardie.fr/ cli/maxsatz2009.c

[18] http://code.google.com/p/relsat

[19] L. Zdeborová and F. Krzakala, arXiv:0902.4185 (2009). 